\title{
Parejas Mixtas de europeos en España: Integración, satisfacción y expectativas de futuro
}

\author{
ÓSCAR A. SANTACREU FERNÁNDEZ \\ IUDESP. UNIVERSIDAD DE ALICANTE \\ FRANCISCO J. FRANCÉS GARCÍA \\ IUDESP. UNIVERSIDAD DE ALICANTE
}

\section{Resumen}

Los matrimonios mixtos son objeto de interés cada vez con más frecuencia. De hecho, dado su constante incremento, estas parejas son uno de los aspectos más visibles del multiculturalismo en España. Este artículo estudia diferentes aspectos referidos a la integración, la satisfacción con la propia vida y las expectativas de futuro en relación a los matrimonios mixtos. Partiendo del modelo de Berry, se emplean los datos procedentes de EIMSS y modelos de ecuaciones estructurales (SEM) sobre los aspectos mencionados, a fin de mostrar el valor central que, a efectos explicativos, tienen en el modelo las parejas mixtas.

Palabras Clave: matrimonios mixtos, multiculturalismo, integración, Modelos de ecuaciones estructurales

\begin{abstract}
Mixed marriages are more and more frequently object of interest. In fact, due to the increase in the number of mixed marriages, they are one of the most visible aspects of multiculturalism in Spain. This article takes into consideration the aspects of integration, overall satisfaction with life and future plans, and puts them in relation to mixed marriages. Starting from and adaption of the Berry's model, we use data from the EIMSS survey and Structural Equation Models (SEM) on the aspects mentioned, in order to show the central value of mixed couples to explain them.
\end{abstract}

Keywords: mixed marriages, multiculturalism, integration, Structural Equation Models 


\section{Introducción}

Es difícil entender el actual contexto español sin tener en cuenta la multiculturalidad y transculturalidad que deriva del hecho de la creciente concentración y diversidad de la población extranjera en España. Si nos fijamos específicamente en las migraciones europeas, comprobamos que tras la incorporación a la Comunidad Europea las importantes barreras a la movilidad laboral intraeuropea que existían para España desaparecen. A pesar de las predicciones de oleadas masivas de inmigración económica desde los países del sur de Europa hacia países del Europa Occidental y Europa del Norte, sucedió lo contrario: los países europeos del sur y de "cohesión" pasaron, en general, de ser países de emigración neta a ser países de inmigración (Mansoor y Quillin, 2007). Así sucedió con España, convertida recientemente en país de inmigración. A raíz de este cambio, y al aumento también de las migraciones extraeuropeas, la sociedad española cuenta ahora con una mayor presencia de grupos de personas unidos por características étnicas, religiosas, lingüísticas y nacionales propias, dando lugar al hecho social denominado multiculturalidad (Touraine A., 1995).

Uno de los aspectos de mayor visibilidad en este sentido es el aumento en el número de matrimonios mixtos, entendidos como aquellos en los que uno de los miembros de la pareja tiene una nacionalidad diferente a la del otro miembro. La principal consecuencia es, sin duda, la formación de familias multiculturales. No se trata de una cuestión sin importancia: la conformación de una pareja de personas pertenecientes a culturas distintas pone en juego muchos aspectos como los sociales, culturales, institucionales, de convivencia, el papel de la familia, el plurilingüismo, la religión, etc. (Observatorio Madrid entre dos orillas, 2005).

En el Anuario Estadístico de Extranjería de 20061 podemos comprobar cómo en el año 2005 se celebraron en España 29.925 matrimonios que entrarían dentro de la definición de matrimonio mixto que hemos expuesto. Estamos hablando nada más y nada menos que del $14,29 \%$ del total de los matrimonios celebrados ese año

\footnotetext{
${ }^{1}$ Secretaría de Estado de Inmigración y Emigración, 2006.
} 
(209.415). De hecho, el número de matrimonios celebrados en España en los que al menos uno de los contrayentes era de nacionalidad extranjera se ha incrementado del 4\% al 14\% entre 1990 y 2004 (Cortina, C.; Cabré, A. y Esteve, A., 2006).

La distribución de estos matrimonios en España viene determinada por la mayor o menor concentración geográfica de la población extranjera en determinadas comunidades autónomas, siendo mayor la concentración en el arco mediterráneo.

Sin duda, en el seno de estas parejas mixtas pueden encontrarse diferencias culturales, posibles conflictos, transmisión de valores culturales e identitarios, etc. En cierto sentido, podríamos hablar de diversas brechas culturales a través de las cuales se ponen en contacto civilizaciones diferentes, cosa que no ocurre, sin embargo, con las familias conformadas por españoles y europeos (Zabalo Escudero E., 2000). De hecho, los colectivos de migrantes procedentes de la Unión Europea no presentan estrategias de acercamiento intercultural a la realidad española. Algunos autores señalan incluso el proceso inverso (García Castaño, F. J. y Granados Martínez, A., (2002). En todo caso no se pueden constatar grandes problemas de adaptación o integración, como constata por ejemplo el hecho de que los hijos de los extranjeros europeos son escolarizados en las escuelas española sin grandes problemas (Pulido Moyano, R. A., 1996).

En este artículo nos centraremos en las parejas mixtas de ciudadanos europeos en España. Específicamente, vamos a analizar los aspectos más relacionados con la integración, la satisfacción general con la vida y los planes de futuro. Partiremos también de una visión amplia de las parejas mixtas, no limitada únicamente a las parejas formalizadas mediante matrimonio legal.

En cuanto a la integración, consideraremos el análisis bidimensional en el que Berry (1980) incluyó el mantenimiento o no de la identidad étnica del inmigrante así como su actitud integradora con el nuevo grupo étnico para explicar el proceso de aculturación. En su modelo se obtenían cuatro orientaciones de aculturación en función de las respuestas a cada una de las dimensiones citadas. Si mantenía sus propios valores étnicos y a la vez tenía relaciones favorables con otros grupos étnicos, se consideraba que tenía una estrategia de integración. Si mantenía sus valores étnicos pero no se relacionaba con otros 
grupos encontramos la orientación hacia la separación o segregación. Si se relacionaba bien con otros grupos a costa de no mantener su identidad étnica estaríamos delante de un caso de asimilación. Finalmente, si no mantiene sus valores étnicos ni relaciones con otros grupos étnicos, estaríamos ante un caso de marginalización.

De acuerdo con Carrascosa González, J. (2002), un extranjero casado con un español o española, residiendo de manera legal e integrada, manifiesta una "doble vinculación" con España, cuyo objetivo radica en el "enraizamiento del extranjero en la sociedad española" y no en la mera "estancia física" del mismo en España. Partiendo de esta observación y del análisis de Berry, vamos a definir al migrante integrado como aquel que se siente próximo tanto a su país de origen como a su actual país de residencia (España), tal y como se muestra en la siguiente tabla:

Tabla 1. Adaptación del modelo bidimensional de Berry

\begin{tabular}{|c|c|c|c|}
\hline & \multicolumn{2}{|c|}{ Se siente del país de origen } \\
\hline & & SÍ & $\mathrm{NO}$ \\
\hline \multirow[t]{2}{*}{ Se siente del país de destino } & SÍ & Integración & Asimilación \\
\hline & $\mathrm{NO}$ & Segregación & Marginalización \\
\hline
\end{tabular}

\section{Planteamiento de un modelo explicativo}

A partir de esta breve introducción, planteamos en este artículo un modelo explicativo que trata de analizar las diferencias que en cuanto a la integración, sentimiento de discriminación y visión de futuro en el país puede plantear el hecho de formar parte de una pareja mixta.

Para ello utilizaremos datos procedentes de la encuesta EIMSS (European Internal Mobility Social Survey), construida en el contexto del proyecto de investigación PIONEUR2 del V Programa Marco de la Unión Europea. En dicho proyecto se realizó un muestreo aleatorio telefónico a ciudadanos de la Unión Europea residentes en países

2 The PIONEUR Project: Pioneers of Europe's Integration "from Below": Mobility and the Emergence of European Identity among National and Foreign Citizens in the EU. European Comission: Fifth Framework Programme, Key Action "Improving the Socio-economic Knowledge Base". Website obets.ua.es/pioneur 
diferentes de su país de origen, en concreto en cinco países (Alemania, Francia, Inglaterra, Italia y España), buscando en cada país específicamente ciudadanos de las otras cuatro nacionalidades implicadas, a fin de identificar los perfiles específicos de las migraciones internas europeas. Se realizaron 250 entrevistas telefónicas para cada nacionalidad en cada uno de estos países, 5000 entrevistas en total.

En este artículo utilizaremos únicamente los datos recogidos en España, lo que supone una muestra de 1000 encuestados en cuotas equidistribuidas por nacionalidad (franceses, ingleses, italianos y alemanes).

A partir de los datos de la EIMSS, hemos seleccionado una serie de variables en función de su capacidad explicativa y de su relación con las parejas mixtas. Así, contaremos con las siguientes variables explicativas:

- La edad del entrevistado en el momento de la migración.

- El hecho de haber vivido previamente en el país de destino o en un tercer país.

- El conocimiento del idioma español previo al momento de la migación.

- El sexo del entrevistado.

- La cualificación del entrevistado.

A la hora de operativizar el concepto de "cualificado" con fines estadísticos, se suele usar el nivel de logros educativos. Así, Liebig, T. y A. Sousa-Poza han definido "cualificado" como "igual a 1 si el encuestado tiene un título universitario o un título de formación profesional y 0 en caso contrario" (Liebig, T.; Sousa-Poza, A., 2005). Sabour considera además la experiencia laboral a la hora de definir a una persona como "cualificada", quedando por tanto su definición "una persona que tiene un nivel educativo mínimo de licenciatura en un determinado campo científico e intelectual con unos cuantos años de experiencia laboral" (Sabour, 1997). Ateniéndonos a la naturaleza de los datos que pretendemos analizar, consideraremos aquí como cualificados a los encuestados con estudios universitarios. 
Junto a estas variables explicativas tendremos otras a explicar y que van a interaccionar entre sí. Tomaremos en primer lugar como posible hecho diferenciador el hecho de formar parte de una pareja mixta y lo pondremos en relación con:

- La integración, entendida tal y como se ha explicado anteriormente.

- El grado de satisfacción general con la vida.

- El sentimiento de discriminación.

- La previsión del futuro vital a largo plazo, entendida como la visión del entrevistado sobre su probable país de residencia cuando alcance la edad de la jubilación.

Teniendo en cuenta que todos los miembros de la muestra son extranjeros franceses, ingleses, alemanes e italianos viviendo en España, nuestra principal hipótesis consiste en que el hecho de pertenecer a una pareja mixta tiene un valor central a la hora de explicar tanto el grado general de satisfacción con la vida como el planteamiento de un futuro a largo plazo en España, aspectos ambos mayores, en términos de cambio medio, para los miembros de parejas mixtas.

Por otro lado el formar parte de una pareja mixta estaría favorecido por el hecho de ser joven en el momento de migrar, tener un mayor conocimiento previo del idioma que favorezca la interacción necesaria en el inicio de cualquier relación, y tener experiencias previas de movilidad en otros países y por tanto posiblemente una mayor competencia intercultural. El sexo del entrevistado también tendrá capacidad explicativa sobre esta variable, como veremos.

A su vez, el planteamiento del futuro va a estar explicado, además de por formar parte de una pareja mixta, por la satisfacción general con la vida y por una menor cualificación del encuestado (entendemos que los cualificados vienen a España principalmente por motivos de trabajo y tenderán a abandonarla al terminar su etapa laboral). El hecho de plantearse un futuro estable en España, y también el hecho de haber vivido en este país previamente, favorecerán la adopción de una estrategia de integración en los términos en los que se ha explicado anteriormente. 
El grado de integración va a influir positivamente en la satisfacción general, junto con el sentimiento de discriminación (explicado a su vez por una menor edad en el momento de la migración y por la capacidad de comparación que da el haber vivido previamente en terceros países) y el pertenecer a una pareja mixta.

De modo resumido podemos presentar el modelo teórico que proponemos en el siguiente diagrama causal (Fig. 1).

Fig. 1. Diagrama causal del modelo propuesto

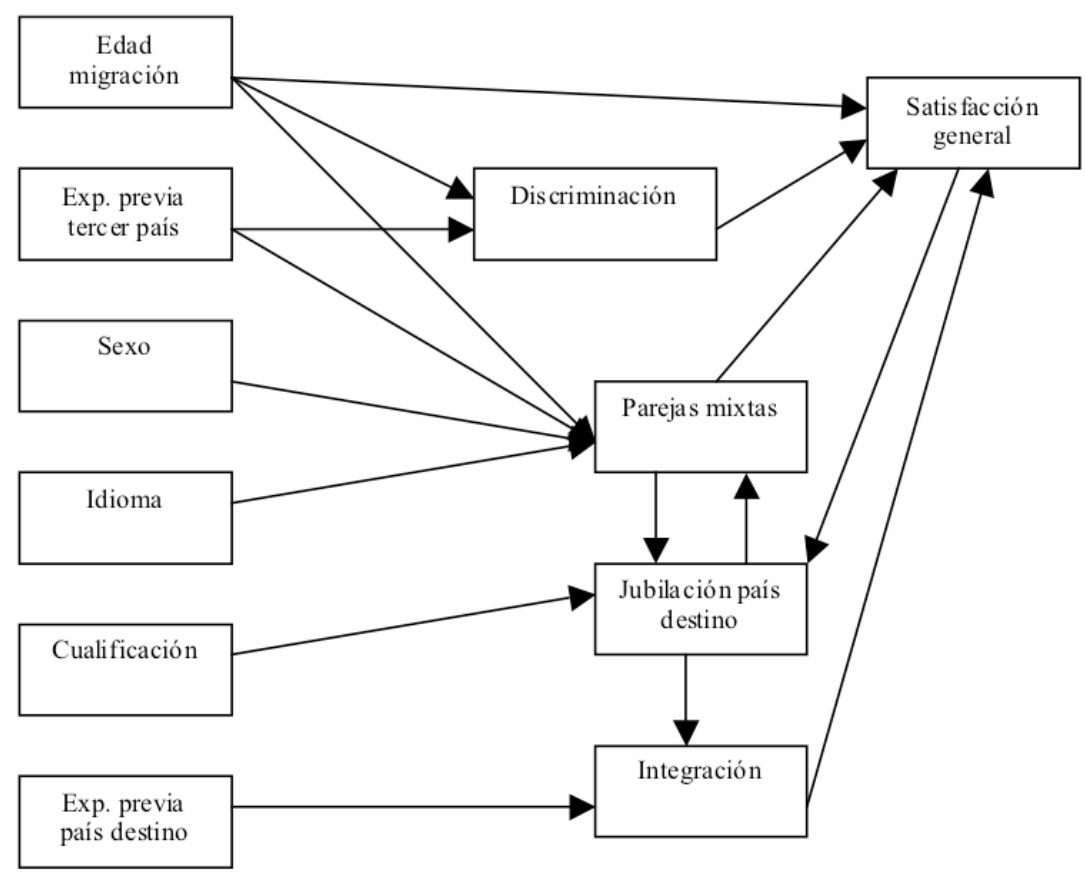

\section{Análisis de los datos}

En primer lugar procedemos a un análisis descriptivo de los datos de la EIMSS, centrándonos básicamente en las variables que formarán parte del modelo. De este modo extraemos las siguientes consideraciones sobre la muestra, que recordemos se circunscribe a ciudadanos ingleses, franceses, italianos y alemanes residentes en España: 
- El grado de cualificación de estos ciudadanos queda reflejado en el hecho de que el $10,2 \%$ de la muestra son migrantes cualificados, es decir, con estudios universitarios.

- Los encuestados presentan una relativamente alta experiencia de movilidad previa: el 20,1\% vivió anteriormente en España y el $37,8 \%$ ha tenido experiencias previas de movilidad en terceros países.

- En relación a esta gran movilidad, un 13,6\% de los encuestados tenía un conocimiento del lenguaje alto o muy alto antes de su migración. El conocimiento actual del idioma español es aún mayor, aumentando el porcentaje de conocimiento alto o muy alto hasta el $52,6 \%$.

- Un alto porcentaje de los encuestados añora de alguna manera su país. Si bien el porcentaje es menor al referirnos a aspectos concretos como la cultura, la comida, el clima, etc, el porcentaje de respuestas afirmativas alcanza el $71 \%$ si nos referimos a echar de menos en general a su país de origen.

- Sólo el 21,2\% puede considerarse integrado en la sociedad española según la adaptación del modelo de Berry presentado anteriormente, en términos de mantenimiento del sentimiento de apego tanto al país de origen como al país de destino.

- El 16,8\% manifiesta haberse sentido discriminado en España por su condición de extranjero.

- Con todo, parece que España convence a sus visitantes, dado que el $46,1 \%$ de la muestra piensa que pasará su período de retiro laboral en España en lugar de volver a su país.

Centrémonos ahora en las parejas mixtas, que suponen el $27,8 \%$ de la muestra. En primer lugar hemos de aclarar que estamos considerando como pareja mixta aquella en la cual el encuestado manifiesta que su pareja tiene una nacionalidad distinta de la suya, independientemente de si dicha pareja es española (si tuviéramos en cuenta sólo los migrantes con pareja española, el porcentaje se reduciría ligeramente hasta el 21,1\%). En todo caso, los encuestados que pertenecen al grupo de parejas mixtas son principalmente franceses (el 33,7\% de ellos son parejas mixtas) e italianos (53,3\%), como puede comprobarse en el siguiente gráfico: 
Fig. 2. Porcentaje de parejas mixtas por nacionalidad del encuestado

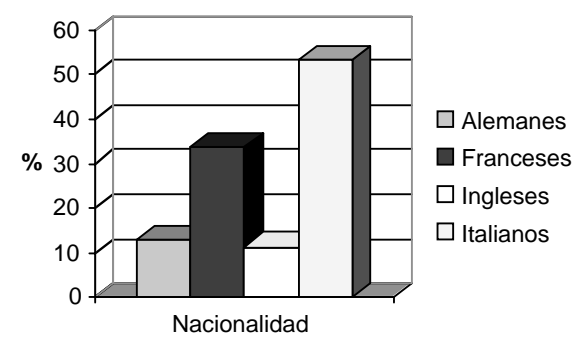

Al aplicar el mismo análisis descriptivo realizado anteriormente, esta vez tomando como factor distintivo el hecho de pertenecer a una pareja mixta, observamos que no existe ninguna diferencia significativa en cuanto al grado de cualificación, el sentimiento de añoranza o la integración del encuestado. Sí encontramos diferencias significativas, en cambio, en otros aspectos:

- Al hablar de la movilidad previa comprobamos que, en el caso de las parejas mixtas, el porcentaje de experiencias previas de movilidad en España es del $26,2 \%$, porcentaje significativo si lo comparamos con el correspondiente al resto de los encuestados $(17,8 \%)$. Esta diferencia es aún mayor al hablar de las experiencias previas de movilidad en terceros países, donde encontramos un $53,3 \%$ en las parejas mixtas frente al $31,9 \%$ del resto.

- El conocimiento previo del idioma español es también significativamente mayor en el caso de las parejas mixtas. Comparadas con el resto de la muestra, encontramos que el $23,3 \%$ de los encuestados con pareja de otra nacionalidad (mayoritariamente española) tenía un nivel alto o muy alto de conocimiento previo del español, frente al 9,8\% del resto de los encuestados. Evidentemente, la diferencia es también observable si hablamos del conocimiento actual del idioma: $79,6 \%$ en parejas mixtas frente al $42,3 \%$ en el resto.

- El grado percibido de discriminación es ligeramente mayor en el caso de las parejas mixtas $(22,6 \%$ frente a $14,6 \%)$, más 
sensibles ante las posibles actitudes discriminatorias de una sociedad en la que se sienten en principio más integrados.

- En todo caso, los encuestados de parejas mixtas creen que pasarán su jubilación en España en un porcentaje mayor $(53,6 \%)$ que el correspondiente al resto de encuestados $(43,2 \%)$.

Este breve análisis exploratorio parece corroborar el modelo explicativo que hemos planteado antes. En todo caso, para determinar en qué grado los datos sustentan las hipótesis de partida utilizamos la metodología SEM (Structural Equation Models, modelos de ecuaciones estructurales). Esta técnica estadística multivariante se utiliza habitualmente para estudiar y analizar las relaciones de dependencia, en términos de carga explicativa, que existen entre las variables que forman parte de un fenómeno social determinado. Como método de análisis, esta técnica define un modelo como un conjunto de ecuaciones estructurales que podemos representar mediante un diagrama causal. A continuación se ofrece el diagrama causal que recoge la explicación en conjunto (Fig. 3), incorporando los coeficientes de ajuste.

Fig. 3. Diagrama causal con coeficientes de ajuste

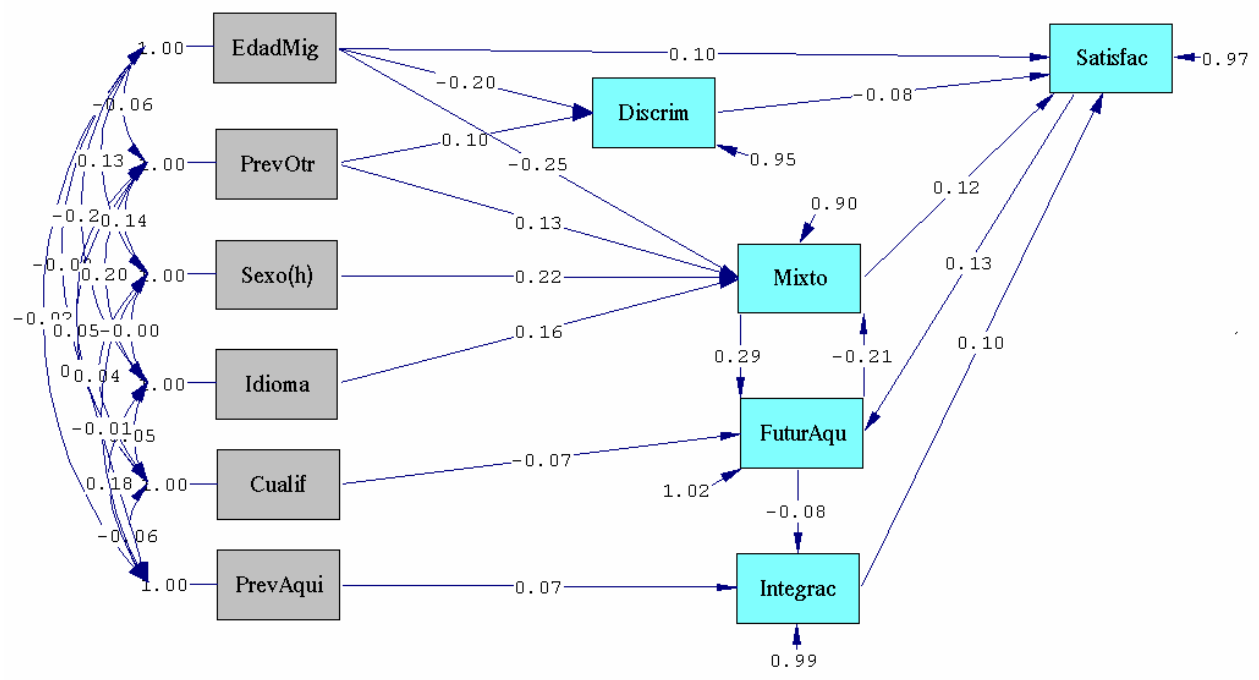

Chi-Square $=27.84, d f=24, P-v a l u e=0.26700$, RMSEA $=0.013$ 
Los pesos de las relaciones vienen expresados en valores estandarizados. Todas las relaciones propuestas y que aparecen en el diagrama causal son estadísticamente significativas, con valores $\mathrm{T}$ superiores a $|2|$. Los estadísticos de bondad de ajuste también indican el buen ajuste del modelo, tal y como se presenta en la tabla 2 .

Tabla 2. Estadísticos de bondad de ajuste del modelo propuesto

\begin{tabular}{|llll|}
\hline Estadísticos & Variación & $\begin{array}{l}\text { Valores } \\
\text { recomendables }\end{array}$ & $\begin{array}{l}\text { Valores } \\
\text { modelo }\end{array}$ \\
\hline RMSEA & & $<0.05$ & 0.013 \\
\hline P- Value & $0-1$ & $>0.05$ & 0.26700 \\
\hline GFI & $0-1$ & $>0.90$ & 0.99 \\
\hline AGFI & $0-1$ & $>0.90$ & 0.99 \\
\hline NFI & $0-1$ & $>0.80$ & 0.95 \\
\hline NNFI & $0-1$ & $>0.95$ & 0.98 \\
\hline
\end{tabular}

Adicionalmente, el Chi-Square de 27,84 es significativo para 24 grados de libertad.

\section{Conclusiones}

Tal y como se observa, el análisis corrobora las hipótesis presentadas anteriormente. En primer lugar, es evidente el valor central en términos explicativos que tiene el formar parte de una pareja mixta. Esta circunstancia, favorecida por una menor edad en el momento de la migración, una mayor experiencia migratoria, un mayor conocimiento del idioma español antes de establecer su residencia en este país y por ser de sexo masculino, explica de forma significativa el hecho de que los encuestados consideren la posibilidad de no volver a sus países de origen al llegar a la edad de la jubilación, tanto de forma directa como a través de una mayor satisfacción general con sus vidas actuales. Naturalmente, cuando afirmamos que las parejas mixtas se plantean pasar su jubilación en España no estamos diciendo que sean los únicos. De hecho, la gran mayoría de quienes consideran que pasarán su jubilación en España no son parejas mixtas, y de ahí la relación negativa que aparece en este sentido en el diagrama. Hemos de

${ }^{3}$ Corresponde a los valores recomendados por los creadores del software LISREL (www.ssicentral.com), ampliamente utilizado en los modelos de ecuaciones estructurales. 
considerar que España, al igual que Francia, recibe principalmente migrantes europeos de edad avanzada. De hecho, la mayor parte de los ciudadanos europeos que pasan su jubilación fuera de su país de origen están en España (Braun, M. y Arsene, C., 2006). Dentro de esta cadena explicativa, observamos que quienes se plantean su futuro en España no mantienen con tanta fuerza, en términos de cambios medios, su apego a su país de origen, lo que influye también en dicho nivel de satisfacción. El grado de cualificación influye también en la consideración de la posibilidad de pasar la jubilación en España, si bien esta relación es negativa debido a dos factores: en primer lugar, tal como se dijo anteriormente, responde a la motivación principalmente laboral de los más cualificados y a su consecuente retorno al país de origen al final de la etapa laboral, y en segundo lugar, al hecho también comentado de que la migración al sur de Europa (Italia y España) está dominada principalmente por migración de retiro. España recibe de hecho a los migrantes de más edad y con menor cualificación, en particular desde Alemania y Gran Bretaña, con baja integración (Ibid, 2006). En todo caso el grado de integración es mayor si ya se estuvo previamente en España, algo lógico si consideramos que ello permite un mayor conocimiento de la cultura y de los valores de los españoles.

En este modelo, el sentimiento de discriminación no muestra ninguna relación explicativa directa y estadísticamente significativa con el fenómeno de las parejas mixtas, sino que va a depender de una menor edad en el momento de la migración y de una mayor experiencia migratoria previa. Con todo, este sentimiento de discriminación estaría ligado a una lógica menor satisfacción general con la vida, y este grado de satisfacción influirá sin duda en el posible planteamiento de una futura jubilación en España. En todo caso, queda claro que, a pesar de los cambios de contexto social que supone el estar residiendo en un país diferente al de origen y los consiguientes posibles conflictos de identidad debidos a la disonancia cognitiva (Rother, N.; Nebe, T., 2006), las parejas mixtas constituyen un espacio que favorece una mayor satisfacción general con la vida y el planteamiento de su futuro en España, si bien no puede demostrarse de forma estadísticamente significativa, al menos en la muestra analizada, ningún efecto directo sobre el grado de integración entendido como el mantenimiento del sentimiento de apego tanto a su país de origen como a su actual país de residencia. Este aspecto es, sin embargo, por su importancia, merecedor de futuros análisis. 


\section{Bibliografía}

Berry, J. W. (1980). Acculturation as varieties of adaptation. In A. Padilha (Ed.), Acculturation: Theory, models and some new findings (pp. 9-25). Boulder, CO, USA: Westview.

Braun, M.; Arsene, C. (2006). The Demographics of Movers and Stayers in the European Union. Final Conference PIONEUR Project, Firenze, March 2006. http://www.obets.ua.es/pioneur/resultados.php

Carrascosa González, J. (2002): "Matrimonios de conveniencia y nacionalidad española", Anales de Derecho, Número 20, 2002. Págs.7-34, Universidad de Murcia.

Cortina, C.; Cabré, A. y Esteve, A.(2006): ¿Con quién se unen los Latinoamericanos en España? Respuestas a partir de tres fuentes estadísticas, II Congreso de la Asociación Latinoamericana de Población: La demografía latinoamericana del siglo XXI, Guadalajara, México, 3 - 5 de Septiembre.

Liebig, T. and A. Sousa-Poza (2005), "Taxation, Ethnic Ties and the Location Choice of Highly Skilled Immigrants", OECD Social Employment and Migration Working Papers, No. 24, OECD Publishing.

M'Hammed Sabour (1997) "The North African intellectual diaspora in the nordic countries. The socio-political factors of expatriation". In M'hammed Sabour \& Knut Vikor Ethnic Encounter and Culture Change. Papers from the Third Nordic Conference on Middle Eastern Studies. Hurst \& Co Publishers. London.

Mansoor A, Quillin B. (2007) Migration and Remittances: Eastern Europe and the Former Soviet Union January. The World Bank

[Observatorio Madrid entre dos orillas]; Centro de Estudios Económicos Tomillo (CEET), S.L y Fundación Directa (2005): Matrimonios mestizos en la Comunidad de Madrid, Estudio 2005, en http://www.entredosorillas.org/

[Observatorio Valenciano de las Migraciones] (2007) - Fundación CeiMigra: Miradas sobre la Inmigración, Movimiento Natural de la Población Inmigrante en la Comunidad Valenciana, Documento Número 6, en: www.ceimigra.net 
Pulido Moyano, R.A. (1996): Los extranjeros en la mente de los niños. Discurso y contexto en la construcción infantil de alteridad, Tesis Doctoral. Departamento de Antropología y Trabajo Social Universidad de Granada.

Rother, N.; Nebe, T. (2006): The Question of European Identity. Final PIONEUR conference, Florence, March 10, 2006. http://www.obets.ua.es/pioneur/resultados.php

Secretaría de Estado de Inmigración y Emigración. Anuario estadístico de Inmigración 2006. Ministerio de Trabajo e Inmigración. Gobierno de España.

http://extranjeros.mtas.es/es/general/DatosEstadisticos_index.html

Touraine A.,(1995):“¿Qué es una sociedad multicultural?”, Claves de Razón Práctica no 561995 p.14.

Zabalo Escudero, E., (2000): "Efectos del matrimonio y sociedad multicultural", Estatuto personal y multiculturalidad de la familia, Madrid, pp.9 a 25. 